\title{
Untangling the History of M31 with Planetary Nebulae
}

\author{
Bruce Balick \\ Astronomy Dept, University of Washington \\ Seattle WA 98195-1580, USA \\ email: balick@uw.edu
}

\begin{abstract}
Recent studies of planetary nebulae and deep color-magnitude diagrams in M 31 have unveiled some unusual star-formation events beyond its spiral arms into its outer regions extending to $100 \mathrm{kpc}$ in projected distance. In this paper I summarize these observations. I shall also highlight some special interpretive surprises suggested by observations of PNe related to their $\mathrm{O} / \mathrm{H}$ abundances, ages, and the luminosities on $\mathrm{PNe}$ well beyond the inner disk.
\end{abstract}

Keywords. planetary nebulae: general; galaxies: individual (M 31), haloes

\section{Introduction}

For many decades M 31 was considered a large, sedate spiral galaxy since the numbers of luminous H II regions in its disk are less than those in the Milky Way (Azimlu et al. 2011) and its $\mathrm{H} \alpha$-to-stellar luminosity ratio is 76 times smaller than its nearby spiral companion M33 (Kennicutt 1988). Recent studies of planetary nebulae ("PNe") and deep color-magnitude diagrams ("CMD") have unveiled some unusual star-formation events beyond its spiral arms $\left(R_{\text {gal }}>17 \mathrm{kpc}\right)$ into its outer regions extending to $100 \mathrm{kpc}$ in projected distance. In this paper I summarize these observations. I shall also highlight some special interpretive surprises suggested by observations of $\mathrm{PNe}$ related to their $\mathrm{O} / \mathrm{H}$ abundances, ages, and the luminosities on PNe well beyond the inner disk.

The bulk distributions of stars in the bulge, inner disk, and halo of M 31 are completely normal for a galaxy of its type (Courteau et al. 2011). For our purposes we note that the large-scale properties of the vast majority of halo stars (stellar types, metalicities, kinematics, and ages) are normal (e.g., Chapman et al. 2006; Koch et al. 2008; Richardson et al. 2009).

\section{Historical context}

Two major spectroscopic surveys of PNe in M31 had been conducted prior to 2000 by Richer, McCall, \& Stasinska (1998), and Jacoby \& Ciardullo (1999), hereafter JC99. Both of these focussed on the chemical abundances of PNe in M31's bulge. JC99 summarized the combined results. Although the average abundances of bulge PNe are not markedly different from those of a handful of disk PNe in their sample, the dispersion of abundances in bulge PNe spans more than a decade. However, neither survey noted any odd trends or behaviors in the total of four disk PNe or the bulge in the combined sample, two of them at $R_{\text {gal }} \sim 14 \mathrm{kpc}$. These results stand today.

So the results of spectroscopic surveys of more than two dozen PNe beyond $17 \mathrm{kpc}$ (Merrett et al. 2006, hereafter "M+06"; Kwitter et al. 2012; Kwitter et al. 2013; Balick et al. 2013; Fang et al. 2013; Corradi et al. 2015, hereafter "C+15") came as a surprise. 
This topic and these papers will dominate my review of the past decade's research on PNe in M31.

This story begins in 2001 with the recognition that a vast extended stellar disk and various stellar streams are superimposed on M31's outer halo (Ibata 2001; Ibata et al. 2005; see also discussions in Hammer et al. 2010 and Ibata et al. 2014). The presence of streams is generally attributed as the outcome of many historical mergers with small satellite galaxies with miscellaneous impact parameters (Font et al., 2006; Fardal et al. 2008, and many subsequent papers). However, the accumulated influence of these numerous, small-mass mergers on the recent star-formation history, the bulk structure, and kinematics of M31 seemed insignificant.

\section{The past decade}

In $2006 \mathrm{M}+06$ published the results of an extensive survey of about $3300 \mathrm{PNe}$ in M31 using high-spatial and spectral resolution studies of their [O III $] \lambda 5007$ lines. After discarding those PNe associated with M 31's small companion galaxies and stellar streams, they analyzed the luminosities, spatial distributions, and kinematics of the 2615 remaining PNe, most of which lie in M31's inner disk. Of special interest in this presentation are those $\mathrm{PNe}$ that lie outside the inner disk. $\mathrm{M}+06$ noted that dozens of the outer PNe were highly luminous, which seemed problematic if all luminous PNe are the descendants of young and massive main sequence stars. (For reference, JC99 noted that $\leqslant 1 \%$ of Galactic PNe lie in the halo.) M+06 also found that despite their halo locations most of the outer PNe share the kinematics of the $\mathrm{H}$ II regions of the inner disk and the $\mathrm{H} \mathrm{I}$ rotation curve (Braun 1991) out to about $R_{\text {gal }} \approx 30 \mathrm{kpc}$. This result was confirmed by Kniazev et al. (2014). This seemingly placed them in the extended stellar disk, hereafter the "exodisk", noted by Ibata et al. (2005).

Shortly after the PN survey was published, our group (alphabetically Balick, Corradi, Henry, and Kwitter) initiated a spectroscopic survey of the outer PNe of M31 with the initial goal of extending measurements of $\mathrm{O} / \mathrm{H}$ gradients (seen in bright disk $\mathrm{H}$ II regions in the disk) to far larger radii. This group had been active in such studies of the Milky Way; however, a paucity of PNe in the Galactic halo as well as highly uncertain distances restricted their gradient studies to $R_{\text {gal }} \approx 18 \mathrm{kpc}$ (Henry et al., 2010). As noted earlier, the M+06 survey had uncovered PNe in M31 out to $R_{\text {gal }} \approx 100 \mathrm{kpc}$ (assuming, as their kinematics suggest, that they are part of the exodisk). So M31 held an opportunity that the Milky Way did not.

The history of M31's star formation in its outer regions abruptly became interesting as the past decade opened.

In 2007 we began a spectroscopic survey of 25 outer and relatively bright PNe from the M+06 survey. We consistently used standard observing and data reduction methods (Henry et al., 2004) so trends are relatively free of systematic bias. Our abundance results also agree with entirely independent measurements of three PNe in the outer northern spur of M31 by Fang et al. (2013). As summarized by C+15, 22 of the total sample have kinematics that place them in the exodisk. Of these, the average $\mathrm{O} / \mathrm{H}$ abundance ratio is $2 / 3$ of solar, with no discernible gradient between $20<R_{\text {gal }}<100 \mathrm{kpc}$. Since PNe inherit their oxygen from the ISM from which they formed, they have an excellent memory of the initial abundances in their birth medium. To the extent that $[\mathrm{O} / \mathrm{H}]$ and $[\mathrm{Fe} / \mathrm{H}]$ are correlated (see JC99), this places the metallicity of the ISM from which these PNe came at least 0.5 dex above those of halo stars and close to that of the two disk PNe in the JC99 survey. It suggests a very different history for the parent stars of the PNe and the other stars in the halo. 
Another major conundrum raised by exodisk PNe is their ensemble age. Spectroscopic observations can be used to place them on an H-R diagram and, so, determine the evolutionary status of their central stars. The exodisk PNe clump around the tracks for final CSPN masses $0.6<M_{*}<0.65 M_{\text {sun }}$. From the initial-final mass relations (e.g., Catalán et al., 2008), the initial stellar masses of the progenitor stars lie between 1.5 and $2.2 M_{\text {sun }}$. Thus the progenitors formed about $3 \mathrm{Gyr}$ in the past. If true, then as a group they originated from a surprisingly metal-rich source long after the halo had formed.

Another conundrum really isn't new. M+06 had noted that the form of the PN luminosity function ("PNLF") of PNe in M31 was uniform, even beyond the spiral arms of M31. The form includes the high-luminosity cutoff at the [OIII] absolute magnitude designated $M^{*}$. Jacoby, Ciardullo, and their collaborators had long established the universal nature of the form of the PNLF in all types of galaxies, including ancient ellipticals.

The first hints of similar properties of stars in one location within the exodisk appeared at the same time (Bernard et al., 2012). Deep CMDs in more than a dozen locations towards the halo of M31 by Bernard et al., (2015a, 2015b) demonstrate that such a starburst occurred and left a substantial residue of $\sim 2$-Gyr-old stars with $[\mathrm{Fe} / \mathrm{H}] \approx 0$ throughout M31's outer regions beyond the HI warp. Using color information Ibata et al. (2014) found metallicity gradients associated with several of the stellar streams outside of M31, though $[\mathrm{Fe} / \mathrm{H}]$ does not exceed -0.5 in any particular region. What's more, using CMDs drawn from the extensive 8-color PHAT survey Williams et al. (2015) uncovered evidence for such a population in the inner disk of M31 as well. Significantly, this starburst is omnipresent in M31.

\section{Conclusions}

In summary, the PNe in the outer parts of M31 have revealed a recent albeit brief attempt at full-body stellar rejuvenation. However, the blush of such a rose rapidly fades. This event might not have been uncovered without luminous PNe outside its inner disk to unveil it. Such events in more distant galaxies can only be uncovered by observing PNe with large telescopes since CMDs are compromised because their stars are inextricably crowded and confused. (It is fortunate that stellar CMDs were able to confirm the PNebased detection of a starburst in M31 and verify the story yielded by PNe.)

What triggered the burst of star formation? McConnachie et al. (2009) have proposed that an M31-M33 gravitational encounter occurred 3 Gy in the past with an impact parameter of $\sim 45 \mathrm{kpc}$. Such an event may have disrupted and redistributed the metal-rich ISM from the inner disk of M31 and generated a burst of star formation throughout M31's inner and outer disks. Although the scenario for the burst of star formation is highly speculative, it remains the most plausible explanation for the existence of the bright PNe throughout M31's huge exodisk. Oddly, however, there is no trace of a corresponding generation of PNe in M33, though a 3-Gyr-old population of stars has been found at one location at the edge of its disk (Bernard et al. 2012).

One strange facet of PNe in M31 is that $M^{*}$ are universal standard candles - even where the stellar populations are largely very old. According to the stellar evolutionary models used by Méndez et al. (2008) for their numerical simulations of the PN luminosity function, and related studies of Schönberner, Jacob, Steffen, and Méndez, the only formation path for $M^{*}$ PNe begins with stars with progenitor masses $>2$ solar masses. Only the rapidly evolving central stars of these PNe can attain the high radiation temperatures needed to ionize [O III] sufficiently quickly, while the emission measure of the expanding nebular gas is still high. However, the final and most puzzling conundrum is the presence of $M^{*} \mathrm{PNe}$ in the ancient stellar populations of bulges and early-type 
galaxies. This challenges our understanding of how geriatric stars evolve (R. Ciardullo, this conference).

\section{Acknowledgement}

I am extremely grateful to my colleagues, Karen Kwitter, Romano Corradi, and Dick Henry, who deserve more credit for their efforts than this review might suggest. All of us appreciate the interest and the feedback of Robin Ciardullo as well as the theoretical work by Roberto Méndez and his colleagues. Finally, I thank Letizia Stanghellini, Miriam Peña, and Roberto Méndez for organizing this productive focus meeting.

\section{References}

Azimlu, M., Marciniak R. \& Barmby, P. 2011, ApJ, 142, 139

Balick, B, Kwitter, K. B., Corradi, R. L. M., et al. 2013, ApJ, 774, 3

Bernard, E. J., Ferguson, A. M. N., Barker, M. K., et al. 2012, MNRAS, 420, 2625

Bernard, E. J., Ferguson, A. M. N., Chapman, S. C., et al. 2015a, MNRAS, 453L, 13

Bernard, E. J., Ferguson, A. M. N., Richardson, J. C., et al. 2015b, MNRAS, 446, 2789

Braun, R. 1991, ApJ, 372, 54

Catalán, S., Isern, J., García-Berro, E., et al. 2008, MNRAS, 387, 1693

Chapman, S. C., Ibata, R., Lewis, G. F., et al. 2006, ApJ, 653, 255

Corradi, R. L. M., Kwitter, K. B., \& Balick, B. 2015, ApJ, 807, 181

Courteau, S., Widrow, L. M., McDonald M., et al. 2011, ApJ, 739, 20

Fang, X., Zhang, Y., Garca Benito, R., et al. 2013, ApJ, 774, 38

Fardal, M. A.; Babul, A., \& Guhathakurta, P. 2008, ApJ, 682L, 33

Font, A. S., Johnston, K. V., Guhathakurta, P., et al. 2006, AJ, 131, 1436

Hammer, F., Yang, Y. B., Wang, J. L., et al. 2010, ApJ, 725, 542

Henry, R. B. C., Kwitter, K. B., \& Balick, B. 2004, AJ, 127, 2284

Henry, R. B. C., Kwitter, K. B., \& Jaskot, A. E. 2010, ApJ, 724, 748

Ibata, R., Chapman, S., Ferguson, A. M. N., et al. 2005, ApJ, 634, 287

Ibata, R., Irwin, M., \& Lewis, G. 2001, Nature, 412, 49

Ibata, R., Lewis, G. F., \& McConnachie, A. W. 2014, ApJ, 780, 128

Jacoby. G, Ciardullo, R. 1999, ApJ, 515, 169

Kennicutt, R. C., Jr. 1988, ApJ, 334, 144

Kniazev, A. Y., Grebel, E. K., Zucker, D. B., et al. 2014, AJ, 147, 16

Koch, A., Rich, R. M., Reitzel, D. B., et al. 2008, ApJ, 689, 958

Kwitter, K. B., Lehman, E. M. M., Balick, B., et al. 2012, ApJ, 753, 12

Kwitter, K. B., Lehman, E. M. M., Balick, B., et al. 2013, ApJ, 768, 97

McConnachie, A. W., Irwin, M. J., Ibata, R. A., et al. 2009, Nature, 461, 66

Méndez, R. H., Teodorescu, A. M., Schönberner, D., et al. 2008, ApJ, 681, 325

Merrett, H. R., Merrifield, M. R., Douglas, N. G., et al. 2006 MNRAS, 369, 120

Richardson, J. C., Ferguson, A. M. N., Mackey, A. D., et al. 2009, MNRAS, 396, 1842

Richer, M., McCall, M. L., \& Stasinska, G. 1998, A\&A, 340, 67

Williams, B. F., Dalcanton. J. J., Dolphin, A. E., et al. 2015, ApJ, 806, 48 